\title{
Prognostic value and functional role of ROCK2 in pediatric Ewing sarcoma
}

\author{
GABRIELA MACIEL VIEIRA ${ }^{1}$, GABRIELA MOLINARI ROBERTO ${ }^{2}$, RÉGIA CAROLINE LIRA ${ }^{3}$, \\ EDGARD EDUARD ENGEL ${ }^{4}$, LUIZ GONZAGA TONE ${ }^{4}$ and MARÍA SOL BRASSESCO ${ }^{5}$
}

\author{
${ }^{1}$ Department of Genetics; ${ }^{2}$ Regional Blood Center; ${ }^{3}$ Department of Biomechanics, Medicine and Rehabilitation of \\ The Locomotor System; ${ }^{4}$ Department of Pediatrics, Ribeirão Preto School of Medicine; ${ }^{5}$ Department of Biology, \\ Faculty of Philosophy, Sciences and Letters at Ribeirão Preto, University of São Paulo, Ribeirão Preto, SP 14040-901, Brazil
}

Received May 25, 2017; Accepted October 25, 2017

DOI: $10.3892 / \mathrm{ol} .2017 .7571$

\begin{abstract}
Ewing's sarcoma (EWS) is a highly aggressive bone cancer that affects children and adolescents. Despite advances in multimodal management, 5-year event-free survival rates for patients presenting with metastases at diagnosis remain at $25 \%$. As key regulators of actin organization, the Rho-associated coiled-coil containing protein kinases, ROCK1 and ROCK2, have been associated with cancer dissemination and poorer prognosis. Recently, in vitro data indicating ROCK2 as a molecular target for the treatment of EWS has been presented. Nonetheless, a deeper exploration of the contribution of this kinase dysregulation in EWS is still necessary. In this regard, the present study aimed to evaluate the expression of ROCK 1 and ROCK 2 in 23 pediatric tumor samples and to verify the prospect of using their pharmacological inhibition through functional assays. Our results showed positive immunostaining for ROCK1 and ROCK2 in the majority samples (75 and 65\%, respectively). A significantly increased risk of incomplete remission in patients with positive immunostaining for ROCK 2 was found $(\mathrm{P}=0.026)$, though no correlations with other prognostic features (huvos classification, FLI1/EWS status, relapse, metastasis or death) were observed. Associations with survival were merely suggestive. Apparent protein expression of both kinases was also found in EWS cell lines (SK-ES-1 and RD-ES). Treatments with selective ROCK inhibitors did not alter cell viability or migration in vitro. However, a significant increase in invasion was observed after treatment with SR3677 (ROCK2 inhibitor) and hydroxyfasudil (pan-inhibitor). Consequently, even though the majority of EWS samples included in our study showed positivity for ROCK1 and ROCK2, the lack of
\end{abstract}

Correspondence to: Professor María Sol Brassesco, Department of Biology, Faculty of Philosophy, Sciences and Letters at Ribeirão Preto, University of São Paulo, 3900 Av. Bandeirantes, Bairro Monte Alegre, Ribeirão Preto, SP 14040-901, Brazil

E-mail: solbrassesco@usp.br

Key words: ROCK, Ewing sarcoma, inhibition, expression, target significant associations with prognosis and absence of appropriate responses to their inhibition in vitro does not support their prospective use as therapeutic targets for the treatment of this metastatic tumor. Larger cohort studies might provide more evidence on whether there is a specific role of ROCK kinases in EWS physiopathology.

\section{Introduction}

Ewing's sarcoma (EWS) is a highly aggressive tumor that occurs mostly in children and young adults and rapidly disseminates to bones, bone marrow, and lungs (1). Despite advances in primary EWS management, the improvement of survival rates for patients with metastases or recurrence has remained modest over the last decades $(2,3)$. Consequently, there is a persistent pursuit for new approaches for its treatment.

On this regard, a recent article by Pinca et al (4) published in Oncology Reports portrayed the effects of ROCKs (Rho-associated coiled-coil containing protein kinases) specific inhibition on the growth, migration and differentiation of two EWS cell lines. The authors showed that exposure of cells to Y27632 (ROCK pan-inhibitor) or SR3677 (ROCK2 inhibitor) significantly reduced migration and growth, while favoring morphology changes and neural differentiation. As a result, the authors embrace the possible use of ROCK2 as a molecular target for the treatment of EWS.

The role of ROCK1 and ROCK2 in cancer cell dissemination through their contribution in actin cytoskeleton organization, cell adhesion and motility has been extensively studied in many tumors of different origins (5-10). However, a deeper appreciation of the role of the dysregulation of these kinases in EWS and their possible associations with patient's prognosis is still indispensable.

\section{Materials and methods}

Clinical samples. Twenty-three consecutive primary EWS tumor samples were obtained by surgeons from the Department of Biomechanics, Medicine and Rehabilitation of the Locomotor System of the Clinics University Hospital (Ribeirão Preto School of Medicine-University of São Paulo) between May 2005 and September 2015. The survival analysis 
was followed until June 2016. No local or systemic treatment had been conducted in these patients before the surgery. All samples were obtained with informed consent and the research approved by the Ethics Committee of the University of São Paulo (no. 43619215.9.0000.5407). Tissues were included in paraffin by the Pathology department of the Clinics University Hospital (Ribeirão Preto School of Medicine, University of São Paulo, Ribeirão Preto, SP, Brazil).

Cell lines and reagents. The EWS cell lines SK-ES-1 and RD-ES were acquired from the Rio de Janeiro Cell Bank (Rio de Janeiro, Brazil). Before the experiments each cell line authentication was conducted in the laboratory of Biochemical Genetics-FMRP/USP, by examining the CSF1PO, D13S317, D16S539, D5S818, D7S820, THO1, TPOX, vWA, and AMEL polymorphic loci for Short tandem repeat profiling (STR) under the supervision of Professor Dr Aguinaldo Luiz Simões. Cells were grown in McCoy's or RPMI medium (Gibco; Grand Island, NY, USA) supplemented with $10 \%$ of fetal bovine serum and an antibiotic mixture (100 units/ml penicillin, and $100 \mu \mathrm{g} / \mathrm{ml}$ streptomycin) and maintained in an incubator at $37^{\circ} \mathrm{C}$ with $5 \% \mathrm{CO}_{2}$ in a humidified atmosphere.

Drug and treatments. The drugs, hydroxifasudil (pan-ROCK inhibitor), GSK429286 and SR3677 (ROCK1 and ROCK2 inhibitor respectively) were purchased from Sigma-Aldrich (St. Louis, MO, USA). For all experiments, the drugs were added on the culture medium immediately before applied to cells. Corresponding control cultures received equal volumes of solvent dimethyl sulfoxide (DMSO).

$R N A$ isolation, reverse transcription and quantitative real-time $P C R$ of $m R N A$. Total RNA from cell lines was extracted using TRIzol Reagent (Invitrogen, Karlsruche, Germany) following the manufacturer's protocol. RNA samples from 9 osteoblast primary cultures were kindly provided by Professor Adalberto Luiz Rosado from the School of Dentistry of Ribeirão Preto, University of São Paulo, and used as controls. The concentration and quality of the RNA was accessed using a ND-1000 NanoDrop spectrophotometer (NanoDrop Technologies; Thermo Fisher Scientific, Wilmington, DE, USA). cDNA was synthetized using the High Capacity kit (Applied Biosystems; Thermo Fisher Scientific, Inc., Waltham, MA, USA) according to the manufacturer's instructions. The qRT-PCR was performed using Taqman ${ }^{\circledR}$ gene assays [ROCK1 (Hs01127699-m1), ROCK2 (Hs00178154-m1)], according to the manufacturer's protocol on the 7500 Real Time PCR System (Applied Biosystems; Thermo Fisher Scientific, Inc.). As reference genes, GUS was used to normalize expression levels. The MRC5 (normal fibroblast) cell line were used as calibrator. Relative expression was calculated by $2^{-\Delta \Delta C T}$ analysis method (11).

Western blot. Total protein was extracted from EWS cell lines with RIPA buffer (Thermo Scientific, Inc.) according to manufacture instructions. Equal amounts of heat-denatured protein samples (50 $\mathrm{mg}$ per lane) were separated on $10 \%$ SDS-polyacrylamide gel electrophoresis and transferred to nitrocellulose membranes (Amersham Pharmacia Biotech, Piscataway, NJ, USA). The antibodies included primary rabbit monoclonal anti-ROCK1 (Ab45171; dilution, 1:500; Abcam, Cambridge, MA, USA), primary rabbit monoclonal anti-ROCK2 (Ab125025; dilution, 1:10,000; Abcam) and rabbit monoclonal anti-GAPDH antibody (AbEPR6256; dilution, 1:10,000; Abcam). The immunoblots were developed using goat anti-rabbit secondary antibody (Ab6721; dilution, 1:5,000; Abcam) followed by detection with the ECL Western Blotting Substrate kit (Abcam, Cambridge, UK) and visualized in a ChemiDoc Bioimaging System (Bio-Rad, Hercules, CA, USA). Expression levels were quantified using Image ${ }^{\circledR}$ software and normalized to loading controls.

Immunohistochemistry. Immunohistochemistry for ROCK1 and ROCK2 was performed in 23 and 22 available tissue samples, respectively (Anti-ROCK1; Ab45171, dilution, 1:250; Anti-ROCK2; Ab125025; dilution, 1:200; Abcam). The reactions were performed with the EXPOSE Mouse and Rabbit Specific HRP/DAB Detection IHC kit (ab80436; Abcam) according to the manufacturer's recommendations. Briefly, histological sections were submitted to xylol and alcohol baths for complete dewaxing and subsequent hydration. Antigen retrieval was performed in steam cooker for $40 \mathrm{~min}$ in Tris-EDTA pH 9.0 buffer. Blocking of endogenous peroxidase and nonspecific binding was performed, respectively, with hydrogen peroxide (15 $\mathrm{min}$ ) and blocking solution of the kit for $10 \mathrm{~min}$. The primary antibodies were diluted as recommended by the manufacturer and incubated at room temperature for $\sim 2 \mathrm{~h}$. After washing, the sections were incubated with the kit complement solution for $10 \mathrm{~min}$ and then with the HRP conjugate for $15 \mathrm{~min}$. Finally, the slides were incubated with diaminobenzidine solution (DAB) for a standard time for each antibody and counterstained with Harris Hematoxylin. A colon adenocarcinoma biopsy was used as a positive control for the anti-ROCK1 antibody, whereas a non-neoplastic kidney sample was used as a control for ROCK2. For negative control of the reactions the primary antibody was replaced with PBS buffer. For evaluation of the immunostaining, the slides were scanned with an Olympus BX61VS Slide Scanner system (Olympus Optical do Brasil Ltda, São Paulo, Brasil) and at least five regions representative of the tumor were analyzed using the IHC Profiler plugin, according to Varghese et al (12). For subsequent statistical analysis, the samples were classified into two groups: Negative or positive (low positive, positive or high positive).

Survival assay. Cell survival assays were performed through the XTT kit (XTT II; Roche Molecular Biochemicals, Mannheim, Germany). In summary, 2,000 cells were seeded in 96-well plates and allowed to attach overnight. Subsequently, cells were treated with different concentrations of each drug and incubated for 48, 72 and $96 \mathrm{~h}$ (h). After treatment, the culture medium was replaced with medium containing $10 \mu \mathrm{l}$ of XTT dye $(3 \mathrm{mg} / \mathrm{ml})$ in each well. The plates were incubated for $4 \mathrm{~h}$ at $37^{\circ} \mathrm{C}$ and the formazan product was measured at 455 and $650 \mathrm{~nm}$ by using an iMarkmicroplate reader (Bio-Rad). As a control, cells treated with the same concentration of drug vehicule, DMSO (Sigma-Aldrich) were used. Each experiment was performed at least in triplicate wells and repeated in three sets of tests.

Colony formation assay. Colony formation assays were performed according to Franken et al (13). Briefly, single 


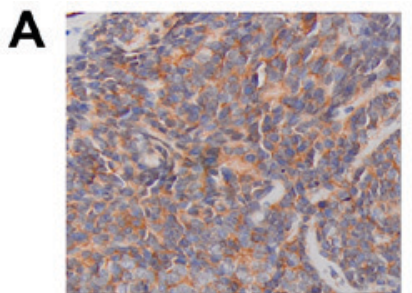

Positive EWS sample

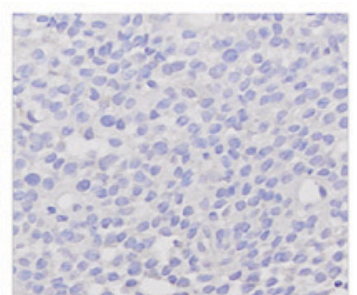

Negative EWS sample

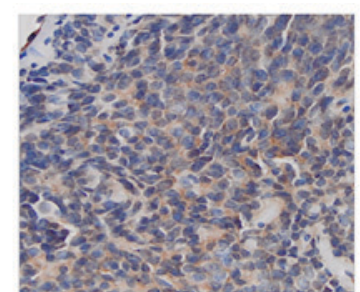

Positive EWS sample

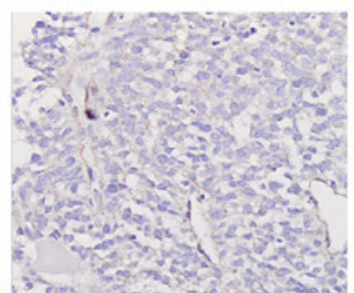

Negative EWS sample
ROCK1

B

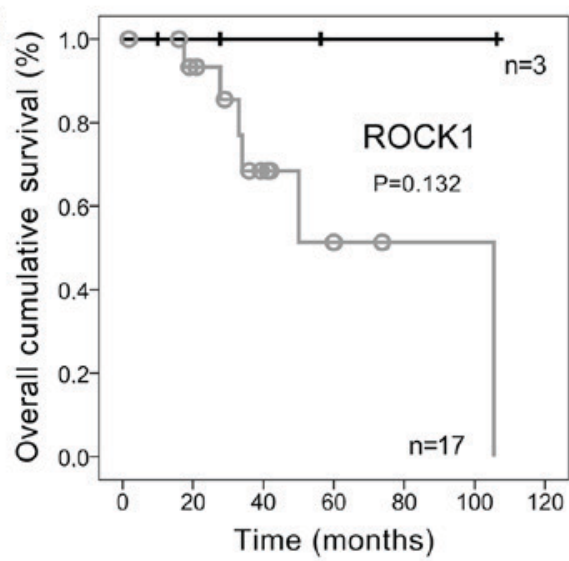

C

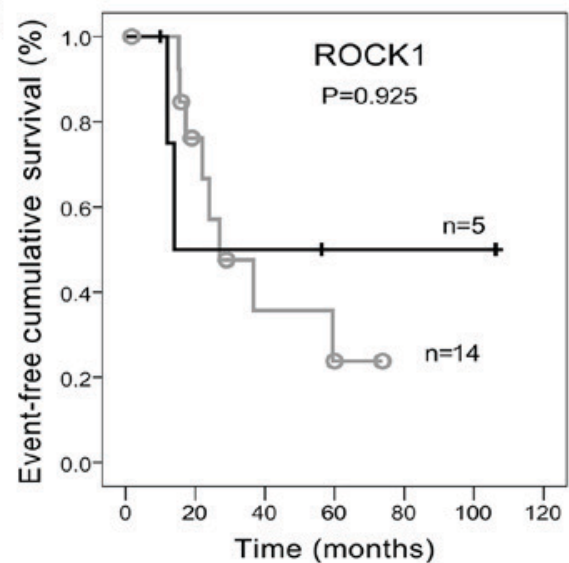

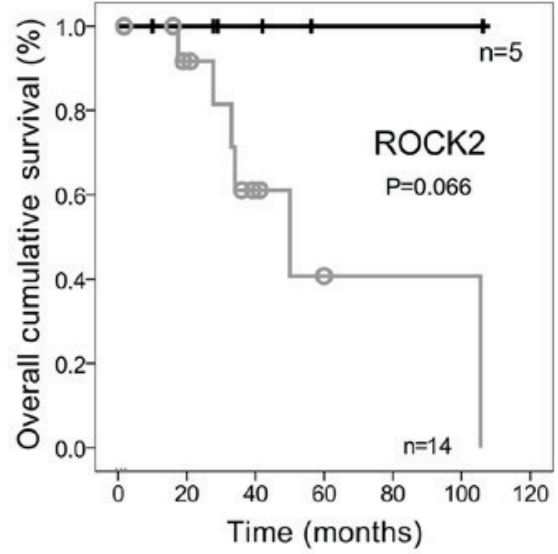

Negative IH

Positive IH

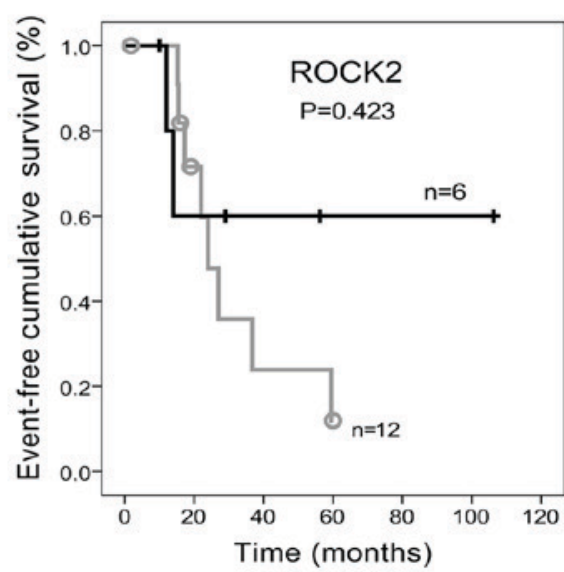

Negative IH

Positive IH

Figure 1. Representative microphotographs of immunodetection staining for ROCK1 and ROCK2 in pediatric EWS and patient's survival curves according to ROCK1 and ROCK2 protein profiles. (A) ROCK1 and ROCK2 positive and negative patterns, respectively. (B) Overall survival curves. (C) Event-free survival curves. Kaplan Meier curves. Log-rank test. Original magnification, x200. ROCK, Rho-associated coiled-coil containing protein kinases; EWS, Ewing's sarcoma.

cell suspensions of 1,000 cells were seeded in 6-well plates and treated with several concentration of each drug for $48 \mathrm{~h}$. After this period, the culture medium were replaced with a drug-free medium and cells incubated at $37^{\circ} \mathrm{C}$ for 7 to 15 days. Then, the colonies were fixed with methanol and stained with Giemsa 3\%. Only colonies containing more than 50 cells were scored. Assays were performed in duplicate in three independent sets of tests.

Cell cycle assay. Cells treated with each drug at different concentrations for $24 \mathrm{~h}$ were detached by trypsin, fixed in $100 \%$ ethanol and stained with propidium iodide for analysis on a Guava Personal Cell Analysis system (Guava Technologies, Hayward, CA, USA) according to the standard protocol provided by the manufacturer. The percentages of cells in G0/G1, S and G2/M phase were analyzed using the GUAVA
Cytosoft software version 4.2.1 (Guava Technologies). All cell cycle assays were performed in triplicate in three independent sets of tests.

Migration assay. Wound healing assays were performed according to Liang et al (14). with minor modifications. Succinctly, cells were grown to confluence on 12-well plates, and scratch wounds were created using a pipet tip $(200 \mu \mathrm{l})$ and photographed at time zero. Then, cells were treated with different concentrations of each drug and cultured for $24 \mathrm{~h}$ in medium with only $1 \%$ of fetal bovine serum. After that period, cells were photographed. The cell-free area was measured with the Motic Images Plus v2.0 software (Motic China Group Co., Ltd., Xiamen, China). Cell migration rates were calculated as the distance travelled by the cells in this area over time. Assays were performed in duplicate in three independent sets of tests. 
Table I. Clinical and pathological features of patients with EWS and corresponding ROCK1 and ROCK2 immunostaining profiles.

\begin{tabular}{|c|c|c|c|c|c|c|c|c|}
\hline \multirow[b]{2}{*}{ Characteristic } & \multicolumn{4}{|c|}{ ROCK1 (n=23) } & \multicolumn{4}{|c|}{ ROCK2 (n=22) } \\
\hline & $\begin{array}{c}(+) \\
\mathrm{n}=17\end{array}$ & $\begin{array}{c}(-) \\
\mathrm{n}=6\end{array}$ & $\begin{array}{l}\text { Odds ratio } \\
(95 \% \text { CI) }\end{array}$ & P-value & $\begin{array}{c}(+) \\
\mathrm{n}=14\end{array}$ & $\begin{array}{c}(-) \\
\mathrm{n}=8\end{array}$ & $\begin{array}{l}\text { Odds ratio } \\
(95 \% \text { CI) }\end{array}$ & P-value \\
\hline \multicolumn{9}{|l|}{ Sex } \\
\hline Male & 8 & 5 & $5.6(0.52-58.91)$ & 0.179 & 7 & 5 & $1.7(0.28-9.82)$ & 0.675 \\
\hline Female & 9 & 1 & & & 7 & 3 & & \\
\hline \multicolumn{9}{|l|}{ Age } \\
\hline$<14$ years & 7 & 2 & $1.4(0.20-9.87)$ & 1.000 & 6 & 3 & $1.2(0.21-7.41)$ & 1.000 \\
\hline$>14$ years & 10 & 4 & & & 8 & 5 & & \\
\hline \multicolumn{9}{|l|}{ Tumor volume ${ }^{\mathrm{a}}$} \\
\hline$>200 \mathrm{~cm}^{3}$ & 7 & 2 & $1.2(0.07-18.35)$ & 1.000 & 7 & 2 & $3.5(0.28-43.2)$ & 0.530 \\
\hline$<200 \mathrm{~cm}^{3}$ & 3 & 1 & & & 2 & 2 & & \\
\hline \multicolumn{9}{|l|}{ Huvos grade ${ }^{a}$} \\
\hline $1-2$ & 5 & 1 & $2.5(0.19-32.19)$ & 0.604 & 4 & 2 & $1.2(0.13-11.0)$ & 1.000 \\
\hline $3-4$ & 6 & 3 & & & 5 & 3 & & \\
\hline \multicolumn{9}{|c|}{ Skeletal location ${ }^{\mathrm{a}}$} \\
\hline Axial & 8 & 2 & $2.0(0.28-14.2)$ & 0.646 & 8 & 2 & $4.8(0.68-33.8)$ & 0.183 \\
\hline Appendicular & 8 & 4 & & & 5 & 6 & & \\
\hline \multicolumn{9}{|l|}{ Remission $^{\mathrm{a}}$} \\
\hline Incomplete & 7 & 1 & $5.2(0.40-68.95)$ & 0.282 & 7 & 1 & $35.0(1.7-702.9)$ & $0.026^{\mathrm{b}}$ \\
\hline Complete & 4 & 3 & & & 1 & 5 & & \\
\hline \multicolumn{9}{|l|}{ EWS/FL1 $1^{a}$} \\
\hline Positive & 9 & 3 & Not calculated & & 8 & 3 & Not calculated & \\
\hline Negative & 1 & 0 & & & 1 & 0 & & \\
\hline \multicolumn{9}{|l|}{ Events } \\
\hline \multicolumn{9}{|l|}{ Metastasis } \\
\hline Yes & 7 & 3 & $0.7(0.11-4.54)$ & 1.000 & 7 & 3 & $1.7(0.28-9.82)$ & 0.675 \\
\hline No & 10 & 3 & & & 7 & 5 & & \\
\hline \multicolumn{9}{|l|}{ Relapse $^{\mathrm{a}}$} \\
\hline Yes & 7 & 1 & $2.3(0.20-27.6)$ & 0.619 & 7 & 1 & $5.8(0.52-64.8)$ & 0.177 \\
\hline No & 9 & 3 & & & 6 & 5 & & \\
\hline \multicolumn{9}{|l|}{ Death $^{\mathrm{a}}$} \\
\hline Yes & 6 & 0 & Not calculated & & 6 & 0 & Not calculated & \\
\hline No & 11 & 4 & & & 8 & 6 & & \\
\hline
\end{tabular}

${ }^{\mathrm{a} C}$ Complete clinicopathological data was not available; 'bignificant P-value (2-tailed Fisher's exact test); EWS, Ewing's sarcoma; ROCK, Rho-associated coiled-coil containing protein kinases; CI, confidence interval.

Invasion assay. Cell invasion was measured by migration of cells through gel-coated Transwell inserts. EWS cells were harvested, re-suspended in serum-free medium, treated with different concentrations of each drug and seeded on the top of Matrigel-coated invasion $8 \mu \mathrm{m}$ pore size chambers (Becton Dickinson \& Co., Franklin Lakes, NJ, USA; 5x10 5 cell/insert). Bellow the insert, the well was filled with medium containing $10 \%$ fetal bovine serum. Cells were then allowed to migrate for $24 \mathrm{~h}$ in an incubator and after that period non-invasive cells were removed from the membrane upper surface with swabs. The ones attached to the lower side of the membrane were fixed with $100 \%$ methanol and stained with Giemsa $3 \%$. The membranes were removed from the inserts, placed on microscope slides with Entelan (Merk, NY, USA) and counted with Image ${ }^{\circledR}$ software in ten random fields at magnification, $\mathrm{x} 20$. Assay was performed in three sets of independent experiments.

Statistical analysis. Associations between ROCK1 and ROCK 2 protein expression and the clinical variables [age ( $<14$ years vs. $>14$ years old); sex (male vs. female); EWS/FLI1 status (positive vs. negative); Huvos grade ( $<90 \%$ of necrotic 

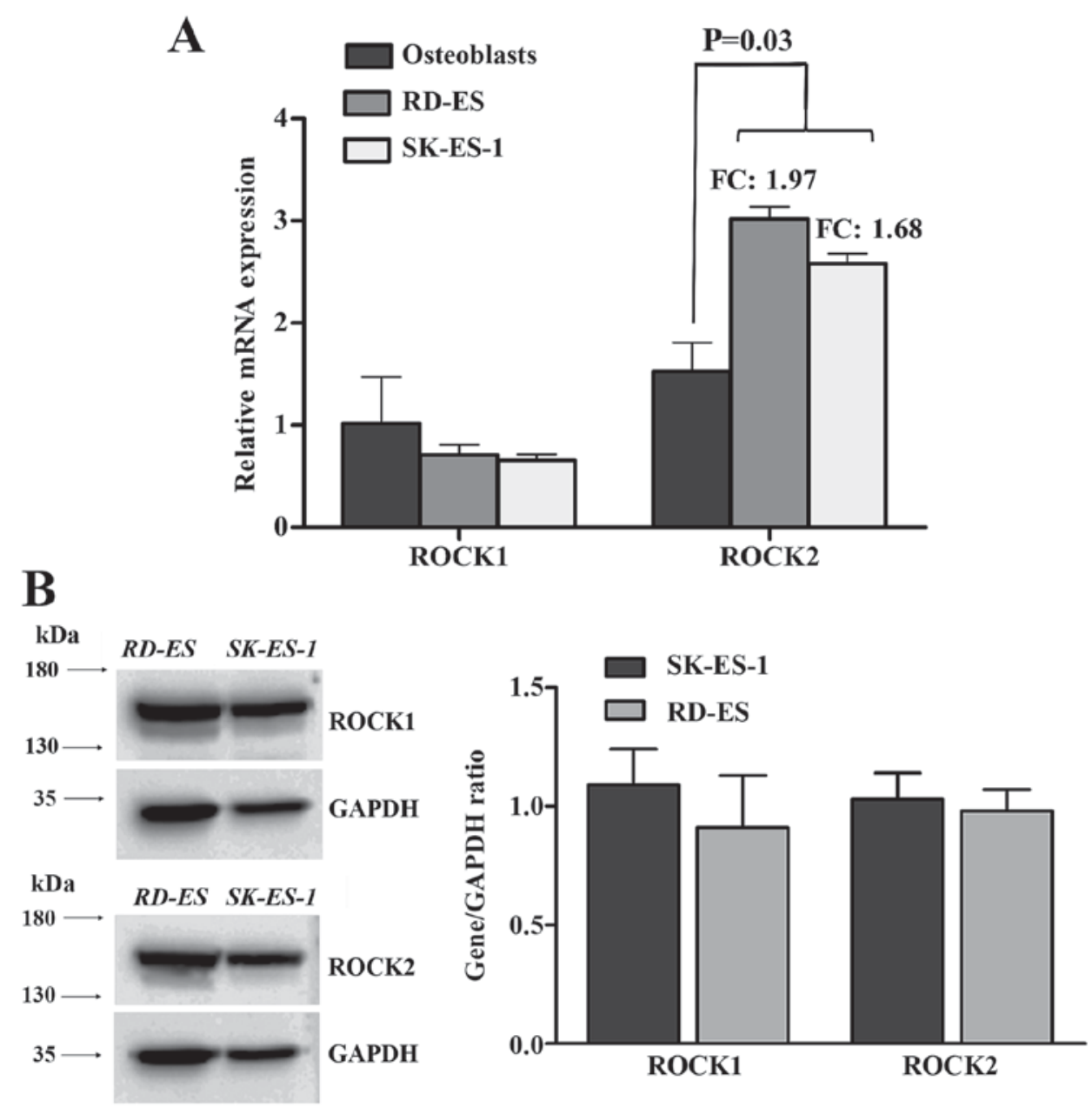

Figure 2. Relative ROCK1 and ROCK2 expression in EWS cell lines. (A) Relative mRNA expression levels of ROCK1 and ROCK2 in primary osteoblast cell lines and the EWS cell lines RD-ES and SK-ES-1. ROCK2, but not ROCK1 was found with significantly higher expression $(\mathrm{P}=0.03)$ when compared to primary osteoblast cell lines with a 1.97 fold-change for RD-ES and 1.68 for SK-ES-1. Each column represents the mean \pm standard deviation. (B) Protein expression levels of ROCK1, ROCK2 and the endogenous GAPDH in SK-ES-1 and RD-ES cell lines. The expression levels of both kinases were found comparable. ROCK, Rho-associated coiled-coil containing protein kinases; EWS, Ewing's sarcoma; FC, fold-change.

areas-Huvos levels 1 and 2 - vs. $>90 \%$ necrotic areas-huvos levels 3 and 4; tumor volume $\left(<200 \mathrm{~cm}^{3}\right.$ vs. $\left.>200 \mathrm{~cm}^{3}\right)$; tumor skeletal location (axial vs. appendicular); remission (incomplete vs. complete); metastasis (presence vs. absence); relapse (presence vs. absence); death (alive vs. deceased] were determined by two-tailed Fisher's exact test. Survival analysis was carried out based on Log-Rank tests represented on Kaplan-Meier curves. The functional assays data was statistically analyzed by Student's two-tailed t-test or One-Way Repeated Measures Analysis of Variance (ANOVA) followed by the Bonferroni Pairwise Multiple Comparison. All tests were carried out for $\alpha=0.05$. All analyses were performed using the SPSS 21.0 software (IBM SPSS, Armonk, NY, USA) and expressed as the mean \pm standard deviation.

\section{Results}

ROCK1 and ROCK2 expression in pediatric EWS. The majority of tumor samples presented positive immunostaining for ROCK1 $(17 / 23 ; 75 \%)$ and ROCK2 (14/23; 65\%) proteins (Fig. 1A). However, no significant associations were observed between their expression and any of the relevant clinical features such as Huvos classification, FLI1/EWS status, relapse, metastasis, or death. Nonetheless, we observed a trend for poorer outcome in patients with positive samples, and significant higher risk of incomplete remission in patients with ROCK2 positive tumors $(\mathrm{OR}=35.0,95 \% \mathrm{CI}, 1.74-702.9 ; \mathrm{P}=0.026)$. In addition, positivity for ROCK 2 seems to indicate increased risk of larger tumor volume $(\mathrm{OR}=2.33,95 \% \mathrm{CI}, 0.22-25.24$; $\mathrm{P}=0.58$ ) (Table I). Moreover, ROCK1 and ROCK2 positivity was also suggestive of lower patient's survival, even though no significant differences were found (Fig. 1B). Event-free survival (EFS) for ROCK1 was estimated at $23.8 \pm 14.1 \%$ for positive samples vs. $50 \pm 25 \%$ for negative ones $(\mathrm{P}=0.925)$. EFS of ROCK2 positive patients was $11.9 \pm 11.1 \%$ vs. $60 \pm 21.9 \%$ $(\mathrm{P}=0.423)($ Fig. 1C).

ROCK2, but not ROCK1 is overexpressed in EWS cell lines. mRNA expression levels of ROCK1 and ROCK2 were evaluated in two EWS cell lines, SK-ES-1 and RD-ES through quantitative real-time PCR. As seen in Fig. 2A, ROCK1 did not show any significant difference in expression when compared to the control (nine primary osteoblast cell lines). Conversely, ROCK 2 was found with significant higher expression $(\mathrm{P}=0.03)$ 
A
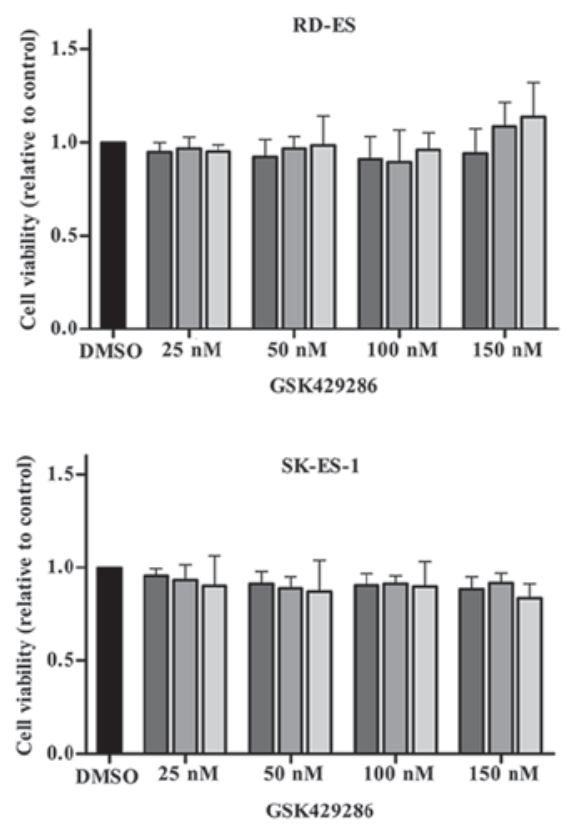

B

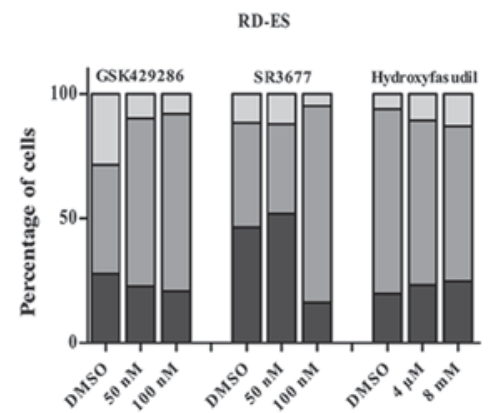

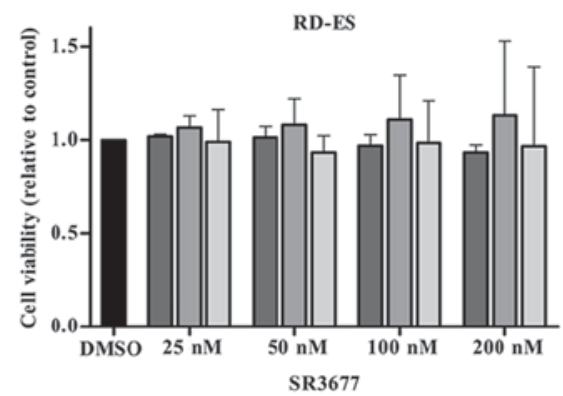

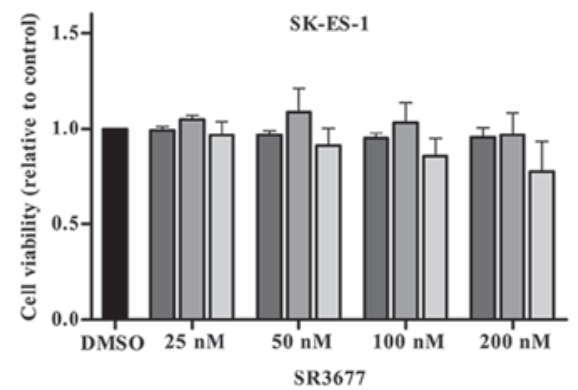

SR3677
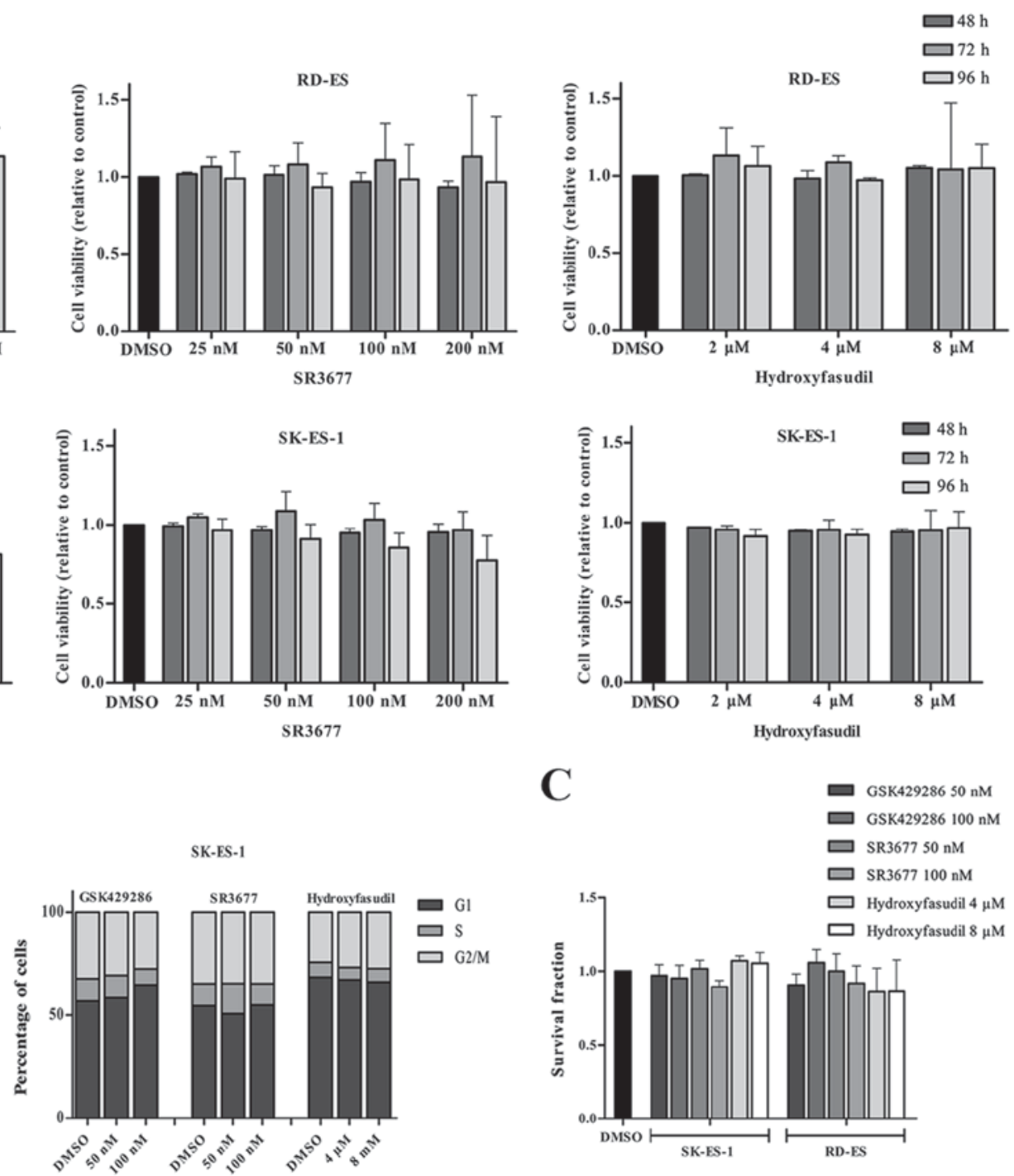

C

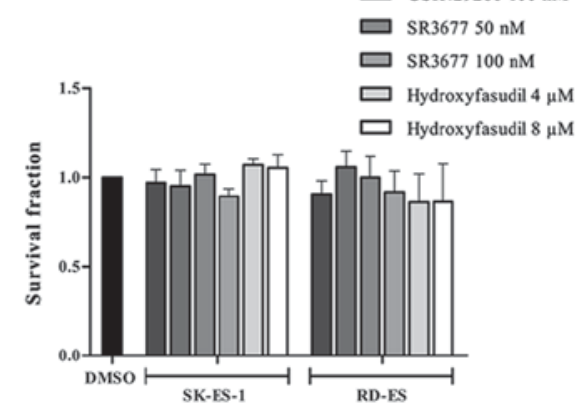

Figure 3. Cell growth of RD-ES and SK-ES-1 cell lines was not affected by ROCK1 and ROCK2 inhibition. (A) Cell viability relative to control (DMSO) after treatment with the drugs GSK429286, SR3677 and hydroxyfasudil for 48, 72 and $96 \mathrm{~h}$ in the RD-ES and SK-ES-1 cell lines. (B) Cell cycle analysis of the RD-ES and SK-ES-1 cell lines after $24 \mathrm{~h}$ of treatment with the drugs GSK429286, SR3677 and hydroxyfasudil. (C) Analysis of the clonogenic capacity of RD-ES and SK-ES-1 cell lines after 48 h of treatment with drugs GSK429286, SR3677 and hydroxyfasudil. Each column represents the mean \pm standard deviation from at least three independent experiments. ROCK, Rho-associated coiled-coil containing protein kinases; DMSO, dimethyl sulfoxide.

compared to controls (fold-change 1.97 for RD-ES and 1.68 for SK-ES-1). Protein expression levels of both kinases were found comparable (Fig. 2B).

Inhibition of ROCK1 or ROCK2 does not show antitumor effects. To investigate the prospect of targeting ROCK1 and ROCK 2 in EWS we evaluated the in vitro effects of three specific inhibitors on cell viability, clonogenicity and cell cycle in the SK-ES-1 and RD-ES EWS cell lines. For each experiment the drugs GSK924286, a specific ROCK1 inhibitor, SR3677, a specific ROCK2 inhibitor and hydroxyfasudil, a pan-ROCK inhibitor were used at different doses according with manufacturer's instructions. Firstly, doses comprising the $\mathrm{IC}_{50}$ reported values were used, being $3.5,7,14$ and $21 \mathrm{nM}$ for GSK924286, $1.25 \mathrm{nM}, 2.5$, 5 and $10 \mathrm{nM}$ for SR3677 and 0.3,0.6, 1.2 and $2.4 \mu \mathrm{M}$ for hydroxyfasudil. Cells were treated for 24, 48, 72 and $96 \mathrm{~h}$ though none of these treatments affected cell viability at any time (data not shown). Then, doses were increased to 25, 50,
100 and $150 \mathrm{nM}$ for GSK924286, 25, 50, 100 and $200 \mathrm{nM}$ for SR3677 and 2, 4 and $8 \mu \mathrm{M}$ for hydroxyfasudil and cells treated for the same periods. Nonetheless, cell viability was not affected again (Fig. 3A). For the other functional assays, doses of $50 \mathrm{nM}$ e $100 \mathrm{nM}$ were chosen for GSK924286 and SR3677 and of 4 and $8 \mu \mathrm{M}$ for hydroxyfasudil. Likewise, and cell cycle dynamics and the clonogenic capacity were not significantly affected by inhibition of ROCKs (Fig. 3B and $\mathrm{C}$ ).

Moreover, the migration capacity of SK-ES-1 cell line was not significantly altered after treatment with any of the drugs as seen through the wound healing assay. Nonetheless, gap closure under treatment with the ROCK2-inhibitor and the pan-inhibitor was increased in $\sim 50 \%$ (Fig. 4A). This effect on the migratory capacity of the cells was evinced by the invasion assays, where treatment with SR377 $50 \mathrm{nM}$ and hidroyfasudil $8 \mu \mathrm{M}$ induced higher penetrance of cells through the Matrigel layer $(\mathrm{P}=0.0063$ and $\mathrm{P}=0.0344$, respectively) (Fig. 4B). 
A

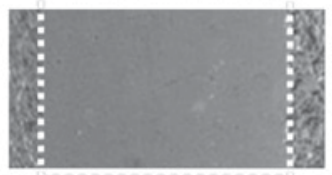

o h

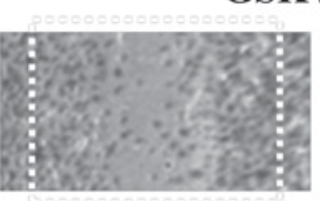

DMSO
GSK429286

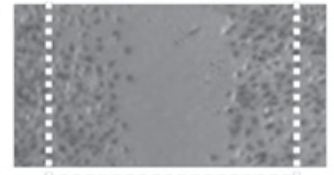

$50 \mathrm{nM}$

SR3677

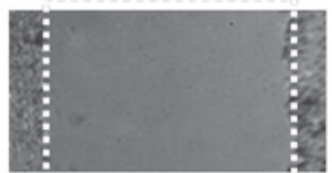

o h

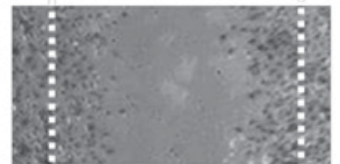

DMSO

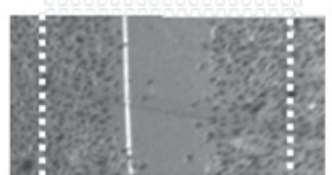

$50 \mathrm{nM}$

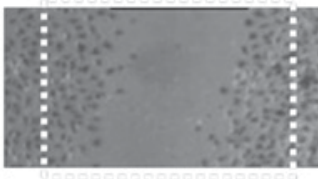

$100 \mathrm{nM}$

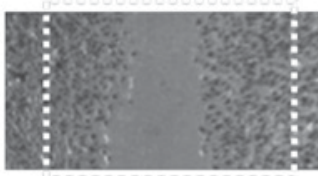

$100 \mathrm{nM}$
Hydroxyfasudil

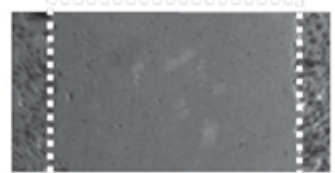

o h

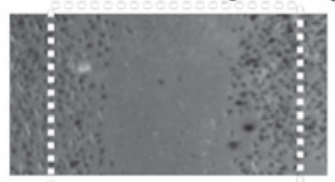

DMSO

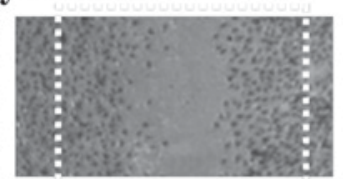

$4 \mu \mathrm{M}$

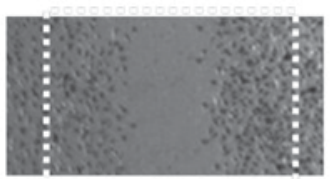

$8 \mu \mathrm{M}$

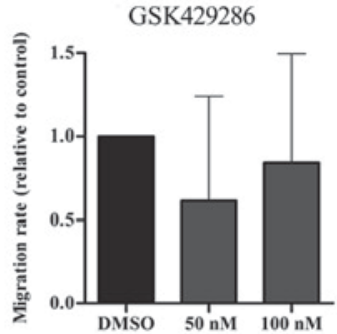

SR3677
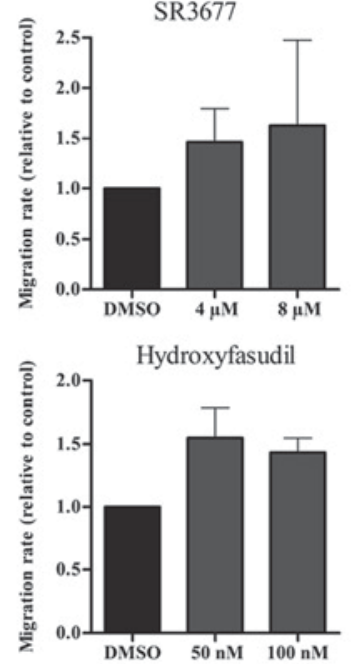

B

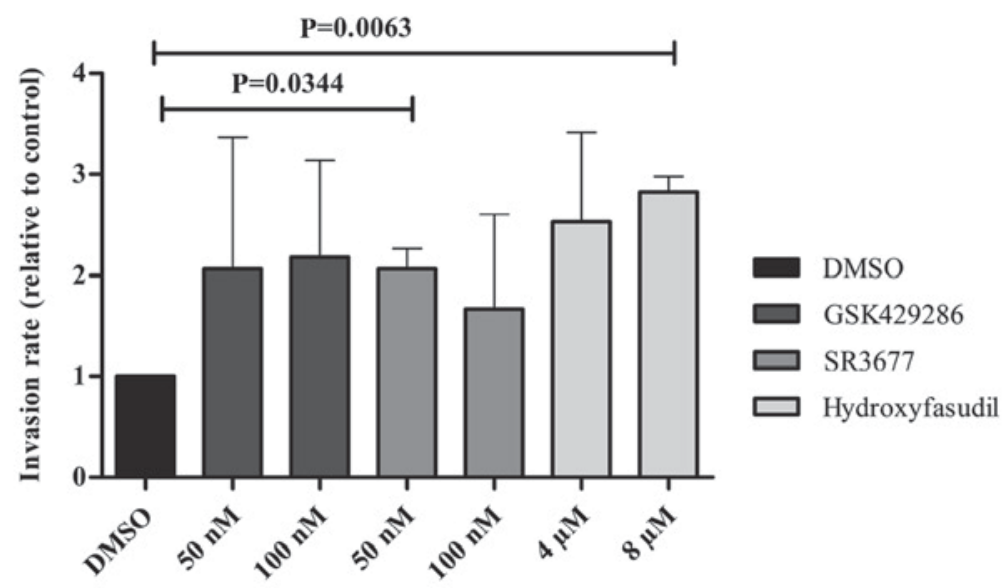

Figure 4. Migration and invasive capacity of the SK-ES-1 cell line was not affected by ROCK1 and ROCK2 inhibition. (A) Migration capacity through wound healing assay at time 0 and after $24 \mathrm{~h}$ of treatment with the control (DMSO) and the drugs GSK429286, SR3677 and hydroxyfasudil. Gap closure was measured through the Motic Images Plus v2.0 software (Motic China Group Co., Ltd.). (B) Invasion capacity through Matrigel assay after 24 h of treatment. Increased invasion was observed after treatment with SR3677 (50 nM) and hydroxyfasudil (8 $\mu \mathrm{M})$ suggesting a stimulating effect after ROCK2 inhibition. The data represents mean \pm standard deviation from at least three independent experiments. Statistical analysis was performed through Student's t-test. $\left({ }^{\#}\right)$ Complete clinicopathological data was not available; (*) significant P-value (Fisher's exact test, 2-tailed). ROCK, Rho-associated coiled-coil containing protein kinases; DMSO, dimethyl sulfoxide.

\section{Discussion}

The Rho-associated kinases ROCK1 and ROCK2 are key regulators of cellular shape and motility by acting on the cytoskeleton $(15,16)$. Over the last decade, their dysregulation has been frequently associated with several carcinogenic and metastasis-related processes such as cell adhesion, migration and invasion $(5,6,10,17)$. Nevertheless, their roles in EWS tumorigenesis/progression and their clinical significance have not been clearly elucidated.

In most tumors studied so far, ROCK1 and ROCK2 are described as oncogenes (7,18-22). Moreover, strong associations between ROCK1 and ROCK2 upregulation and poor prognosis have been described in osteosarcoma, gastric and laryngeal squamous cell carcinoma $(7,20,22)$.

In agreement with these studies, our results showed positive immunostaining for ROCK1 and ROCK2 in the majority of pediatric EWS tumor samples. Furthermore, even though the correlation between patient's survival and ROCK1 or ROCK 2 positivity was only suggestive and there were no associations with clinical features such as HUVOS classification, FLII/EWS status, relapse, metastasis or death, we found a significantly increased risk of incomplete remission in patients 
with positive immunostaining for ROCK2. Nonetheless, these results need to be viewed with caution because of the small number of samples evaluated.

Higher levels of ROCK2 gene expression were also found in EWS cell lines (SK-ES-1 and RD-ES) with conspicuous protein expression of both kinases. In view of these, we tested the prospect of using the pharmacological inhibition of either ROCK through several functional assays in vitro. Three ROCK inhibitors were used: One specific for ROCK1 (GSK429286), one specific for ROCK2 (SR3677) and a pan-inhibitor (hydroxyfasudil).

Initially, we used the doses ranging within the $\mathrm{IC}_{50}$ indicated by the manufacturers, but there were no changes on cell viability after treatment with any of the drugs at such doses. Treatment was also ineffective even after increasing the doses $\sim 10$ times (proliferation and cell cycle). The clonogenic capacity assay, which not only predicts long-term cell viability, but also evaluates the sum of all forms of cell death, also failed to demonstrate any cellular influence irrespective of inhibition of either ROCK1, ROCK2 or both kinases.

Several authors have demonstrated that inhibition of ROCK1 and ROCK2 using other strategies (such as siRNA or microRNAs) causes a decrease in cell invasion and migration in various types of neoplasia $(6,8,9,23,24)$. Similar results have also been reported after treating cells with other inhibitory compounds such as HA-1077 (fasudil), WF-536, Y-27632 and RKI-1447 (8,23,25-28).

The potentiality and selectivity of GSK429286, SR3677, hydroxyfasudil have been repeatedly confirmed in tumors of different origins even using comparable or lower doses than those used in the present study (29-36).

Most recently, Pinca et al (4) performed and in vitro study were they demonstrated ROCK1 and ROCK2 expression in a panel $(n=9)$ of EWS cell lines, and showed that inhibition of these kinases with Y27632 or SR3677 resulted in diminished growth and migration capacity in two EWS cell lines (SK-ES-1 and 6647). However, the inhibitory effects were independent of the protein levels (ROCK1 was $\sim 3 \mathrm{x}$ less expressed in SK-ES-1, for instance). Moreover, individually, the authors showed that ROCK1 expression was higher in the RD-ES cell line whereas ROCK2 expression was higher in the SK-ES-1 cell line, what was not reflected in our study. Similarly, even after treating the same cell line (SK-ES-1) with the same drug (SR3677) functional assays were not reproducible, though Pinca et al (4) used a $\times 100$ higher concentration, which is more than $\mathrm{x} 3,000$ higher than the $\mathrm{IC}_{50}$ reported by the manufacturers. Consequently, their in vitro data might point towards a certain resistance to ROCK inhibition in EWS cells. Of note, it is well established that $>1 \mu \mathrm{M}$ SR3677 acts on several off-target kinases (33) including PKA [which promotes tumor growth and metastasis in EWS (37), MRCK [another mediator of cell contractibility (38) and AKT1 that plays important roles in EWS survival (39).

Moreover, our experiments also showed disparate results on cell migration and invasion which were increased after treatment of the SK-ES-1 cell line with SR3677 and Hydroxyfasudil, suggesting a stimulating effect after ROCK2 inhibition. Abe et al (28) previously reported similar results after treating urothelial carcinoma cells with HA-1077. Likewise, Mertsch and Thanos (40) demonstrated that knockdown of ROCK2 significantly increases the invasive potential of cells in a substrate independent manner.

In this way, even though the majority of EWS samples included in our study showed positivity for ROCK1 and ROCK2, the lack of conspicuous associations with prognosis and absence of effective responses to their inhibition in vitro, do not support their prospect use as therapeutic targets for the treatment of this highly metastatic tumor.

In the future, larger cohort studies might provide more evidence on whether there is a specific role of ROCK kinases in EWS physiopathology.

\section{Acknowledgements}

The authors would like to acknowledge FAPESP for financial support and to thank Mrs. Monica Azevedo de Abreu for technical assistance. FAPESP (Fundação de Amparo à Pesquisa do Estado de São Paulo; grant 2014/03877-3) and VGM fellowship 2014/07118-0.

\section{References}

1. West DC: Ewing sarcoma family of tumors. Curr Opin Oncol 12: 323-329, 2017.

2. Ladenstein R, Pötschger U, Le Deley MC, Whelan J, Paulussen M, Oberlin O, van den Berg H, Dirksen U, Hjorth L, Michon J, et al: Primary disseminated multifocal Ewing sarcoma: Results of the Euro-EWING 99 trial. J Clin Oncol 28: 3284-3291, 2010.

3. Bernstein M, Kovar H, Paulussen M, Randall RL, Schuck A, Teot LA and Juergens H: Ewing's sarcoma family of tumors: Current management. Oncologist 11: 503-519, 2006.

4. Pinca RS, Manara MC, Chiadini V, Picci P, Zucchini C and Scotlandi K: Targeting ROCK 2 rather than ROCK1 inhibits Ewing sarcoma malignancy. Oncol Rep 37: 1387-1393, 2017.

5. Hsu CY, Chang ZF and Lee HH: Immunohistochemical evaluation of ROCK activation in invasive breast cancer. BMC Cancer 15: 943, 2015.

6. Vigil D, Kim TY, Plachco A, Garton AJ, Castaldo L, Pachter JA, Dong H, Chen X, Tokar B, Campbell SL and Der CJ: ROCK1 and ROCK2 are required for non-small cell lung cancer anchorage-independent growth and invasion. Cancer Res 72: 5338-5347, 2012.

7. Zhang J, He X, Ma Y, Liu Y, Shi H, Guo W and Liu L: Overexpression of ROCK1 and ROCK2 inhibits human laryngeal squamous cell carcinoma. Int J Clin Exp Pathol 8: 244-251, 2015.

8. Zhang P, Lu Y, Liu XY and Zhou YH: Knockdown of Rho-associated protein kinase 1 suppresses proliferation and invasion of glioma cells. Tumour Biol 36: 421-428, 2015.

9. Kroiss A, Vincent S, Decaussin-Petrucci M, Meugnier E, Viallet J, Ruffion A, Chalmel F, Samarut J and Allioli N: Androgen-regulated microRNA-135a decreases prostate cancer cell migration and invasion through downregulating ROCK1 and ROCK2. Oncogene 34: 2846-2855, 2015.

10. Sun K, Duan X, Cai H, Liu X, Yang Y, Li M, Zhang X and Wang J: Curcumin inhibits LPA-induced invasion by attenuating RhoA/ROCK/MMPs pathway in MCF7 breast cancer cells. Clin Exp Med 16: 37-47, 2016.

11. Livak KJ and Schmittgen TD: Analysis of relative gene expression data using real-time quantitative PCR and the 2(-Delta Delta C(T)) method. Methods 25: 402-408, 2001.

12. Varghese F, Bukhari AB, Malhotra R and De A: IHC Profiler: An open source plugin for the quantitative evaluation and automated scoring of immunohistochemistry images of human tissue samples. PLoS One 9: e96801, 2014.

13. Franken NA, Rodermond HM, Stap J, Haveman J and van Bree C: Clonogenic assay of cells in vitro. Nat Protoc 1: 2315-2319, 2006.

14. Liang CC, Park AY and Guan JL: In vitro scratch assay: A convenient and inexpensive method for analysis of cell migration in vitro. Nat Protoc 2: 329-333, 2007.

15. Matsumura F: Regulation of myosin II during cytokinesis in higher eukaryotes. Trends Cell Biol 15: 371-377, 2005. 
16. Izawa I and Inagaki M: Regulatory mechanisms and functions of intermediate filaments: A study using site- and phosphorylation state-specific antibodies. Cancer Sci 97: 167-174, 2006.

17. Shi J, Surma M, Zhang L and Wei L: Dissecting the roles of ROCK isoforms in stress-induced cell detachment. Cell Cycle 12: 1492-1500, 2013.

18. Kamai T, Tsujii T, Arai K, Takagi K, Asami H, Ito $\mathrm{Y}$ and Oshima H: Significant association of Rho/ROCK pathway with invasion and metastasis of bladder cancer. Clin Cancer Res 9 : 2632-2641, 2003.

19. Lane J, Martin TA, Watkins G, Mansel RE and Jiang WG: The expression and prognostic value of ROCK I and ROCK II and their role in human breast cancer. Int J Oncol 33: 585-593, 2008.

20. Liu X, Choy E, Hornicek FJ, Yang S, Yang C, Harmon D, Mankin $\mathrm{H}$ and Duan Z: ROCK1 as a potential therapeutic target in osteosarcoma. J Orthop Res 29: 1259-1266, 2011.

21. Babeto E, Conceição AL, Valsechi MC, Peitl Junior P, de Campos Zuccari DA, de Lima LG, Bonilha JL, de Freitas Calmon M, Cordeiro JA and Rahal P: Differentially expressed genes in giant cell tumor of bone. Virchows Arch 458: 467-476, 2011.

22. Wu YJ, Tang Y, Li ZF, Li Z, Zhao Y, Wu ZJ and Su Q: Expression and significance of Rac1, Pak1 and Rock1 in gastric carcinoma. Asia Pac J Clin Oncol 10: e33-e39, 2014.

23. Itoh K, Yoshioka $\mathrm{K}$, Akedo $\mathrm{H}$, Uehata $\mathrm{M}$, Ishizaki $\mathrm{T}$ and Narumiya S: An essential part for Rho-associated kinase in the transcellular invasion of tumor cells. Nat Med 5: 221-225, 1999.

24. Genda T, Sakamoto M, Ichida T, Asakura H, Kojiro M Narumiya S and Hirohashi S: Cell motility mediated by rho and Rho-associated protein kinase plays a critical role in intrahepatic metastasis of human hepatocellular carcinoma. Hepatology 30: 1027-1036, 1999.

25. Takamura M, Sakamoto M, Genda T, Ichida T, Asakura H and Hirohashi S: Inhibition of intrahepatic metastasis of human hepatocellular carcinoma by Rho-associated protein kinase inhibitor Y-27632. Hepatology 33: 577-581, 2001.

26. Nakajima M, Katayama K, Tamechika I, Hayashi K, Amano Y, Uehata M, Goto N and Kondo T: WF-536 inhibits metastatic invasion by enhancing the host cell barrier and inhibiting tumour cell motility. Clin Exp Pharmacol Physiol 30: 457-463, 2003.

27. Patel RA, Forinash KD, Pireddu R, Sun Y, Sun N, Martin MP, Schönbrunn E, Lawrence NJ and Sebti SM: RKI-1447 is a potent inhibitor of the Rho-associated ROCK kinases with anti-invasive and antitumor activities in breast cancer. Cancer Res 72 : 5025-5034, 2012.

28. Abe H, Kamai T, Hayashi K, Anzai N, Shirataki H, Mizuno T, Yamaguchi Y, Masuda A, Yuki $\mathrm{H}$, Betsunoh $\mathrm{H}$, et al: The Rho-kinase inhibitor HA-1077 suppresses proliferation/migration and induces apoptosis of urothelial cancer cells. BMC Cancer 14: 412, 2014

29. Ohnaka K, Shimoda S, Nawata H, Shimokawa H, Kaibuchi K, Iwamoto Y and Takayanagi R: Pitavastatin enhanced BMP-2 and osteocalcin expression by inhibition of Rho-associated kinase in human osteoblasts. Biochem Biophys Res Commun 287: $337-342,2001$
30. Shimokawa H: Rho-kinase as a novel therapeutic target in treatment of cardiovascular diseases. J Cardiovasc Pharmacol 39: 319-327, 2002.

31. Hinderling PH, Karara AH, Tao B, Pawula M, Wilding I and Lu M: Systemic availability of the active metabolite hydroxy-fasudil after administration of fasudil to different sites of the human gastrointestinal tract. J Clin Pharmacol 47: 19-25, 2007.

32. Goodman KB, Cui H, Dowdell SE, Gaitanopoulos DE, Ivy RL, Sehon CA, Stavenger RA, Wang GZ, Viet AQ, Xu W, et al: Development of dihydropyridone indazole amides as selective Rho-kinase inhibitors. J Med Chem 50: 6-9, 2007.

33. Feng Y, Yin Y, Weiser A, Griffin E, Cameron MD, Lin L, Ruiz C, Schürer SC, Inoue T, Rao PV, et al: Discovery of substituted 4-(pyrazol-4-yl)-phenylbenzodioxane-2-carboxamides as potent and highly selective Rho kinase (ROCK-II) inhibitors. J Med Chem 51: 6642-6645, 2008

34. Wang HL, Hu SH, Chou AH, Wang SS, Weng YH and Yeh TH: H1152 promotes the degradation of polyglutamine-expanded ataxin-3 or ataxin-7 independently of its ROCK-inhibiting effect and ameliorates mutant ataxin-3-induced neurodegeneration in the SCA3 transgenic mouse. Neuropharmacology 70: 1-11, 2013.

35. Nichols RJ, Dzamko N, Hutti JE, Cantley LC, Deak M, Moran J, Bamborough P, Reith AD and Alessi DR: Substrate specificity and inhibitors of LRRK2, a protein kinase mutated in Parkinson's disease. Biochem J 424: 47-60, 2009.

36. Chapman S, McDermott DH, Shen K, Jang MK and McBride AA: The effect of Rho kinase inhibition on long-term keratinocyte proliferation is rapid and conditional. Stem Cell Res Ther 5: 60, 2014.

37. Luo W, Xu C, Ayello J, et al: Protein phosphatase 1 regulatory subunit 1A (PPP1R1A) promotes tumor growth and metastasis via inhibition of protein phosphatase 1 in Ewing sarcoma. In: AACR 107th Annual Meeting 2016 American Association for Cancer Research, New Orleans, LA, 2016.

38. Bouin AP, Kyumurkov A, Régent-Kloeckner M, Ribba AS, Faurobert E, Fournier HN, Bourrin-Reynard I, Manet-Dupé S, Oddou C, Balland M, et al: ICAP-1 monoubiquitylation coordinates matrix density and rigidity sensing for cell migration through ROCK2-MRCK $\alpha$ balance. J Cell Sci 130: 626-636, 2017.

39. Hotfilder M, Sondermann P, Senss A, van Valen F, Jürgens H and Vormoor J: PI3K/AKT is involved in mediating survival signals that rescue Ewing tumour cells from fibroblast growth factor 2-induced cell death. Br J Cancer 92: 705-710, 2005.

40. Mertsch S and Thanos S: Opposing signaling of ROCK1 and ROCK 2 determines the switching of substrate specificity and the mode os migration of glioblastoma cells. Mol Neurobiol 49: 900-915, 2014

This work is licensed under a Creative Commons Attribution-NonCommercial-NoDerivatives 4.0 International (CC BY-NC-ND 4.0) License. 